\title{
Automatic Skin Lesions Classification from Dermoscopic Images Employing Deep Learning
}

\author{
Pablo Minango \\ Yuzo Iano \\ Ana Carolina Borges Monteiro \\ Reinaldo Padilha França \\ Gabriel Gomes de Oliveira
}

\section{CITE THIS ARTICLE}

Minango, Pablo; Iano, Yuzo; Monteiro, Ana Carolina Borges; França, Reinaldo Padilha and de Oliveira, Gabriel Gomes; 2019. Automatic Skin Lesions Classification from Dermoscopic Images Employing Deep Learning. ISSN Print: 2446-9246 ISSN Online: 2446-9432. doi: 10.18580/ setijbe.2019.14. Web Link: http://dx.doi.org/10.18580/setijbe.2019.14 


\title{
Automatic Skin Lesions Classification from Dermoscopic Images Employing Deep Learning
}

\author{
Pablo Minango, Yuzo Iano, Ana Carolina Borges Monteiro, Reinaldo Padilha França and Gabriel Gomes de \\ Oliveira
}

\begin{abstract}
Skin cancers are the most incidental in Brazil. Thousands of Brazilians are diagnosed annually with the disease, which occurs due to the abnormal growth of the cells that make up the skin and, therefore, can give rise to several types of skin cancer, being divided into two types melanoma and nonmelanoma. Skin cancer, which represents respectively $25 \%$ of the malignant tumors and about $30 \%$ of the cases of cancer in Brazil, being the majority due to the excessive exposure to the sun's ultraviolet rays. Skin tumors are usually perceived more efficiently, and when diagnosed early, they are more likely to heal. The present study is relies on the development of an algorithm based on Deep Learning for the recognition of tumors in skin images. The AlexNet, which is a Deep Learning architecture is modified to attending our classification problem. The experiments are conducted through 1400 and 2400 images, after twice training with different optimizer, SGD is the better optimizer with $99.79 \%$ of accuracy and $0.0120 \%$ of loss in training, for the scenery of 2400 images.
\end{abstract}

Index Terms-Deep Learning, Python, Biomedical Image Processing, Skin, Melanoma.

\section{INTRODUCTION}

$\mathbf{T}$ HE melanoma skin cancer originates in melanocytes (melanin-producing cells, a substance that determines skin color), which is more common in white adults, appearing anywhere on the body, on the skin or mucous membranes, in the form of spots or signs. On the other hand, black-skinned individuals, it is more common in clear areas such as the palms of the hands and soles of the feet. This type of disease is especially dangerous because it can spread to other tissues of the body, i.e., if not detected early, it generates metastases that make the patient's clinical picture more serious [1][2].

Although skin cancer is the most frequent in Brazil and accounts for about $30 \%$ of all registered malignancies. Melanoma represents only $3 \%$ of malignant neoplasms of the organ, being the most serious type, due to its high possibility of provoking metastasis (spread of cancer to other organs).

Among the symptoms, there are spots on the skin, wounds that do not heal, and spots that change their appearance, and melanomas can appear on healthy skin or existing skin nevi. It may also form brown, irregular, flat or protruding skin plaques with different color spots, or hard black or gray

P. Minango is pursuing an M.Sc. degree in Electrical Eng. (EE), at the Lab. of Vis. Comm. (LCV) at the State Univ. of Campinas (UNICAMP), pablodavid218@gmail.com

Dr. Y. Iano is the LCV-UNICAMP head yuzo@decom.fee.unicamp.br A.C.B. Monteiro is currently pursuing an Ph.D. degree in EE at LCVUNICAMP, monteiro@decom.fee.unicamp.br

R. Padilha is currently pursuing an Ph.D. degree in EE at LCV-UNICAMP, padilha@decom.fee.unicamp.br

G. G. Oliveira is currently pursuing an M.Sc. degree in EE at LCVUNICAMP, oliveiragomesgabriel@ieee.org lumps. Melanomas may vary in relation to their appearance, where some are brownish, flat, irregular plaques and contain small black spots, and others are tall brownish-brown colored plaques with red, white, black or blue dots, where for sometimes melanoma appears as a hard tumor of a red, black or gray color [1][3][4].

The prognosis of this type of cancer can be considered good if it is detected in its initial phase, and in recent years, there has been a great improvement in the survival of patients with melanoma, mainly due to the early detection of the tumor and the introduction of new immunotherapeutic drugs. Still, in consideration, the most common type, non-melanoma skin cancer, has low lethality. However, their numbers are very high. This disease is caused by the abnormal and uncontrolled growth of the cells that make up the skin, where the cells are arranged forming layers, and according to those that are affected, the different types of cancer are defined. The most common types are basal cell and squamous cell carcinomas, where the rarer and lethal than carcinomas, melanoma is the most aggressive type of skin cancer [2][4].

Brazil has an estimated 6260 new cases each year, of which 2920 are men, and 3340 are women (2018 - INCA (National Cancer Institute)), and unfortunately there are 1794 deaths, where 1012 men and 782 women (2015 - SIM (Mortality Information System). Early detection of cancer is a strategy to find a tumor at an early stage and thus enable a greater chance of treatment. This detection can be done through the investigation with clinical, laboratory or radiological examinations, of people with signs and symptoms suggestive of the disease (early diagnosis), or with the use of periodic examinations in people without signs or symptoms (tracing) but belonging to groups with a greater possibility of having such a disease.

Among the main factors, sun exposure ends up being the main means of obtaining the disease, through ultraviolet (UV) rays is a significant risk factor for most melanomas, where they damage the DNA of skin cells and thus affect the control of their cell growth [4].

People who have taken too much sun through their lifetime without adequate protection (sunscreen and/or sunblock) are at increased risk for melanoma, where sunburns, especially in childhood or adolescence, increase the risk of developing skin cancer. Still taking into consideration that geographically, living near the equator or at higher altitude also increases the risk, once the sun's rays are more direct. This people living at high altitudes are more exposed to UV radiation [1][4].

In recent years, we are experiencing a revolution in the area of Artificial Intelligence (AI) [5] - [8] and the main driver of this revolution are technologies based on Deep Learning (DL), 
and taking into account the recent advances in computational power have enabled the possibility of developing algorithms based on Computer Vision and related to DL [5] - [9]. This method, in simple terms, is the part of Machine Learning (ML) that, through high-level algorithms, mimics the neural network of the human brain, generally using deep neural networks and depending on many data for training [10] - [12].

In order to reach the more advanced DL level, the artificial neural networks principle has been developed to support discrete layers, connections and data propagation directions, and such data is subjected to several non-linear processing layers that simulate the way of thinking of neurons [10][13][14][15][16].

In a simplified and generic way, DL is complex algorithms built from a stack of layers of digital "neurons", fed by an immense volume of data, capable of recognizing images and speech, processing them, and learning to perform extremely tasks without human interference, in this scenario having classification and recognition tasks, especially for image recognition [16]-[19].

Python is a language that was created for production and development in an easy, practical and fast way, being an agile, easy and objective language, democratizing its teaching, making it increasingly sought after. Being an object-oriented language, its simplicity is its main characteristic. It is also open and non-profit language.

Pyhton is ideal for scientific applications as DL where it is an expressive language, where it is easy to translate the reasoning into an algorithm, and still taking into account that it allows the work and development with data science and ML [18], [20]-[25].

Thus, in this paper, an algorithm based on DL was developed, which aims to the recognition of skin melanomas patterns, with the use of 1200 and 2400 images for training, wherewith show the accuracy and the loos for each training.

The present paper is organized as follows: Section 2 discusses DL algorithms. Section 3 presents the proposed algorithm methodology. Section 4 presents the results and, finally, in Section 5, the conclusions are discussed.

\section{DEEP LEARNING}

DL is one of the technological trends of the moment, which is ML. ML refers to the fact that computers and devices perform their functions without seeming programmed and as soon as they learn as they are used. DL can be considered as type of ML for the purpose of training computers to perform tasks as humans, i.e., speech recognition, image identification and prediction types, through configurations and standardization, into the set of data that will be worked [18] - [25]

In this way, DL learns alone and is fed by the multiple data generated at all times, through multiple analyzes in the sealed dataset, being able to decipher for example natural language and to relate terms, generating a corresponding meaning, as well as used in image processing and computer vision. Such approaches allow a complex and abstract representation of the data forming an orderly classification, through the use of algorithms that do not require a pre-processing of the data and automatically generate optimal results through the hierarchical layers of representation consisting of the technique, where such layers are non-linear data [16], [18] - [27].

Nowadays, the importance of DL can be seen by a direct connection to neural networks, which provided, for example, the automatic learning used for the creation of autonomous cars as well as the significant improvement of the searches on the web. Further, Deep Learning techniques have been enhancing computers' ability to classify, recognize, detect, and describe them over time in order to understand [18], [20]-[28]. DL and ML are concepts that go hand in hand are closely linked with Artificial Intelligence (AI). They are not synonyms, and DL evolved from machine learning. Since AI is a rather broad term, a branch of computer science, in which research is developed to find solutions that simulate the human capacity for reasoning. ML is already using concepts of AI, through algorithms, making the machines able to organize data and identify patterns without necessarily a pre-programming, making in simple terms. AI is similar to the process of human reasoning, related to this process of learning by inputs, and thus finding patterns. Moreover, finally, there is DL, where it can be considered, to be at a deeper level, with high-level algorithms, where machines begin to act similarly to the neural network of the human brain. Thus, the data are submitted to several layers (in the same way as a network of neurons), in order to recognize images and/or speech. For example, without human intervention in the sequence [22] - [29].

So, essentially, DL is an emerging technological within the field of AI, which is considered a subcategory of ML. DL uses neural networks to improve speech recognition, computer vision, image processing, and natural language processing [23]- [27].

Due to the iterative nature of DL, much computational power is needed to solve problems where its complexity grows as the number of layers employed and consequently, the necessary data volumes are getting large enough to improve the network training. The dynamic nature of DL presents the opportunity to introduce dynamic behaviors to analytics related to the dataset, that is, due to the capacity for continuous improvement and adaptation to changes in the pattern of underlying information [22] - [26].

Technologically speaking, DL performs the "training" of a computational model so that it can decipher and recognize the pattern in the data set where this model relates terms and characteristics, thus resulting in the inference of meaning as it feeds with a large amount of data. DL has many practical applications already in use by companies around the globe, among the many existing techniques we can relate speech recognition and image, image classification, object detection, content description, natural language processing, recommendation systems popularized by Amazon and Netflix, for example where they hit with certain precision the users' interest after taking action on their platform, just as from previous behaviors, systems such as Siri and Cortana are partially fed by DL, is also the base technology for tools like Google Translate, among others [23] - [29].

In short, with an enormous amount of computing power, today's machines have the possibility and ability to recognize 
objects and translate voice in real-time, among many other complex activities of human life, finally making the AI applicable [15]-[26].

\section{Methodology}

Nowadays, pattern recognition and medical images classification using Deep Learning are possible to computational advances and large datasets [30].

Convolutional Neural Networks (CNN), is an architecture of Deep Learning inspired by the human visual cortex. CNN is utilized in several medical application as skins lesion classification problems [31], pancreas segmentation [32], extraction of vessels in fundus images [33], and brain tumor segmentation [34] [35].

In this work, we employ the architecture of AlexNet [36], where has five convolutional layers and three fully connected layers. This architecture is used to obtain the binary classification between benign and malign cancer.

\section{A. Dataset Description}

The International Skin Imaging Collaboration (ISIC) [37] dataset of open access was utilized for training AlexNet architecture. ISIC is an organization that impulses Melanoma Project by providing the access of their images, for academics and industries, with the purpose of decrease mortality rate occasioned for the skin cancer known as Melanoma, because if skin cancer is early recognized in its initial stages, the probability of cure is better.

ISIC is a collector dataset with almost 23906 images of many different skin lesions with their own histopathological diagnostic. Our principal focus is the classification of images with benign and malignant cancer, which represents binary classification.

In this work, AlexNet architecture is trained twice with different quantities of images. This procedure is described below.

\section{B. Architecture Description}

We use the idea of AlexNet [36], which was used and applied to recognize the ImageNet Large Scale Visual Recognition Challenge. Fig 1 depicts the detailed configuration. The structure of AlexNet consists of five convolutional layers, which include the concept of MaxPooling [38] and BatchNormalization [39] in each one of their layers.

The original AlexNet architecture was modified for adapting our problem. The first part of this architecture is the block of input images, determined by the batch size hyperparameter. The output of the first block (IN) passes through five convolutional blocks to obtain the better accuracy during the training classification, where each one of these convolutional blocks are composed for small convolutional filters also named kernels. The size of these kernels are matrices of $(11 \times 11)$ for $\mathrm{C} 1,(5 \times 5)$ for $\mathrm{C} 2$ and $(3 \times 3)$ for $\mathrm{C} 3$, C4 and C5 (see Fig 1). Each one of these blocks is equipped with Rectified Linear Unit (ReLU) as the activation function of each layer.

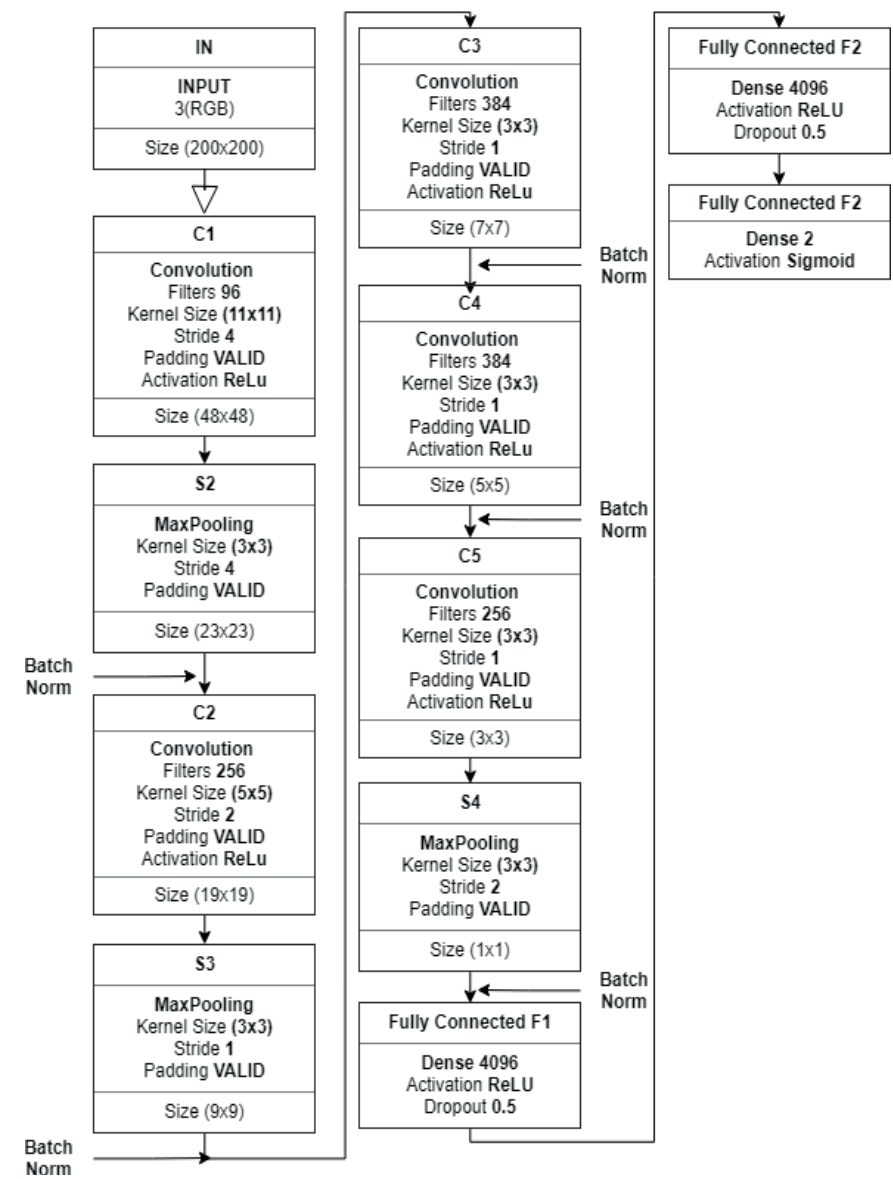

Fig. 1. AlexNet architecture adapted to our problem.

In Fig 1, the last three layers are known as dense layers. During the training step, in each one of the dense layers, every neuron has a probability $p$ of being temporally off. It means that it is deactivated, but probably it will be activated during the next steps. This process is known as Dropout [40].

After each batch size of images are filtered, the results pass to the dense layer stage, which is composed of three dense layers. This work modifies the last one-layer output in order to perform a binary classification between benign and malignant, as well as their activation function was modified from a Softmax to a Sigmoid ${ }^{1}$, which is very used for binary classifications problems.

With the use of AlexNet architecture modified and previously described, the weights of the output are sent to an optimizer during each one epoch, with the purpose of to measure accuracy and loss. The results of these optimizers are tested in each one epoch until attaining their convergence. To attain this premise, we use three different types of optimizer, which are Stochastic Gradient Descent (SGD), RMSprop, and Adam [41]. We consider an initial learning rate of 0.001 for the three optimizers with a Binary cross-entropy loss function [42]. Furthermore, an early stopping criterion was implemented to interrupt the training process after the training accuracy "AUC" does not improve on 10 epochs.

${ }^{1}$ When applied a Sigmoid activation function in the last layer. The architecture only learns to predict values between 0 and 1 . For this reason is one of the most used in binary classification. 


\section{RESUlTS AND DisCUSSION}

We employed the Keras package [43] in order to build and train the model. For data and results visualization, we used the Matplotlib package [44]. All codes were implemented in the Kaggle environment, which provides a free programming environment with the following specifications described in Table I.

TABLE I

KAGGLE ENVIRONMENT SPECIFICATIONS

\begin{tabular}{|l|c|c|}
\cline { 2 - 3 } \multicolumn{1}{l|}{ Specifications } & CPU & GPU \\
\hline Cores & 4 & 2 \\
\hline Gigabytes of RAM & 16 & 13 \\
\hline
\end{tabular}

AlexNet architecture was trained twice times with different quantity of dataset images. During the first training, we choose 1400 images for each class, which is 700 images for benign and 700 images for malignant. All images are resized in $200 \times 200$ pixels RGB and normalized (subtract from the mean and divide by the standard deviation).

For the second training, we considered 2400 images, which are divided into 1200 images of benign and 1200 images of cancer. The same conditions described above were taking in this training.

The evaluation of our methodology was performed using the following metrics in the training dataset:

- Accuracy, which represents the correct predictions in each one class divided by the total number of predictions.

- Loss, which corresponds to the number of images wrong classify, considering the true label.

Fig 2. shows the accuracy and the loss of our methodology for the first training. The SGD optimizer outperforms the Adam and RMS optimizer due to SGD achieves a percentage of $97.66 \%$ and $0.0567 \%$ in learning and loss, respectively.

Table II, shows the comparison performance of the three optimizers.

TABLE II

ACCURACY AND LOSS COMPARISONS OF THE DIFFERENT TYPES O OPTIMIZERS.

\begin{tabular}{|l|c|c|c|c|c|c|}
\cline { 2 - 7 } \multicolumn{1}{c|}{} & Adam & Epochs & RMSprop & Epochs & SGD & Epochs \\
\hline Acc & 96.66 & 19 & 87.64 & 46 & $\mathbf{9 7 . 6 6}$ & 34 \\
\hline Loss & 0.0817 & 19 & 0.3141 & 46 & $\mathbf{0 . 0 5 6 7}$ & 34 \\
\hline
\end{tabular}

For the second training, we can appreciate the performance of the model in Fig 3. Again, in this case, SGD is better over the others optimizers achieved a $99.79 \%$ of learning and $0.0120 \%$ of loss.

Table III shows the performance of the three optimizers, where we appreciate that SGD has better accuracy and loss results.

Concerning the epochs of each one optimizer, Adam and RMSprop were stopped with the criterion of early stopping because they attain an accuracy convergence, and do not improve more if we continued with more epochs of training.

\section{CONCLUSION}

We have proposed a binary classification to detect the early stages of melanoma. The solution used was AlexNet

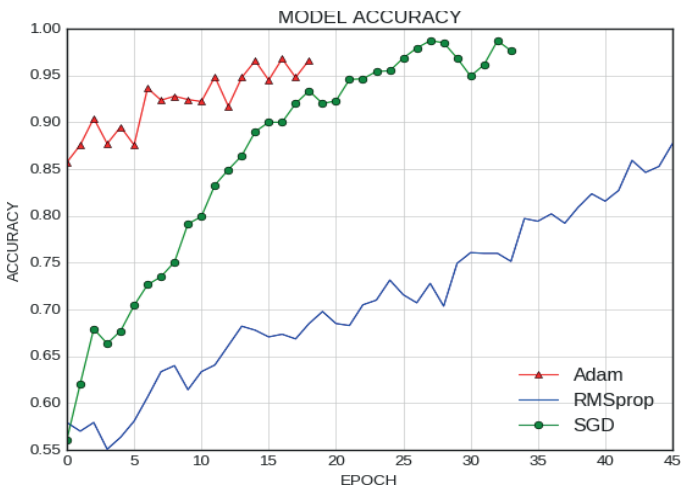

(a)

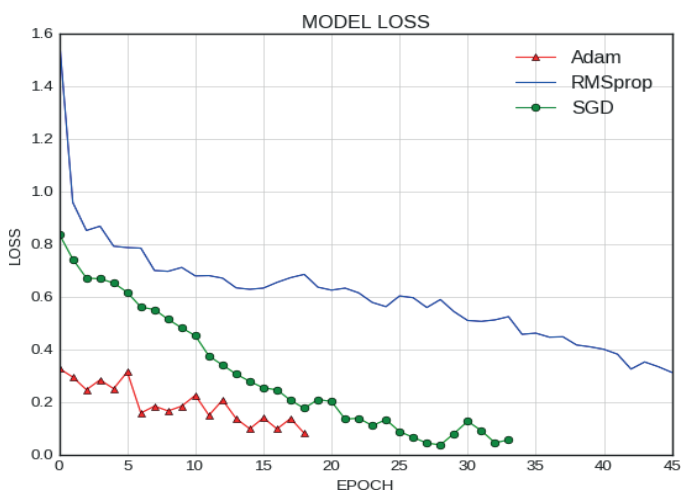

(b)

Fig. 2. Performance of the model with 1400 training images. (a) Accuracy, (b) Loss

TABLE III

ACCURACY AND LOSS COMPARISONS OF THE DIFFERENT TYPES OF OPTIMIZERS.

\begin{tabular}{|l|c|c|c|c|c|c|}
\cline { 2 - 7 } \multicolumn{1}{c|}{} & Adam & Epochs & RMSprop & Epochs & SGD & Epochs \\
\hline Acc & 97.85 & 24 & 89.72 & 27 & $\mathbf{9 9 . 7 9}$ & 35 \\
\hline Loss & 0.0591 & 24 & 0.2434 & 27 & $\mathbf{0 . 0 1 2 0}$ & 35 \\
\hline
\end{tabular}

which is an architecture of learning. We have tested it with different quantities of images for three types of optimizers, where we have observed that SGD is the better optimizer with an accuracy of $99.79 \%$ for 2400 images of training. In this context, we have appreciated the improvement of learning with the increasing of the quantity of images. In ouradaptation of AlexNet, the number of epochs does not have a difference when analyzed SGD optimizer since for 1400 images SGD needs 34 epochs for obtaining its maximum accuracy which was $97.66 \%$ and for 2400 images it needs 35 epochs, for obtaining an accuracy of $99.79 \%$.

\section{ACKNOWLEDGMENT}

We thank the State University of Campinas (UNICAMP), Brazil for providing all the necessary infrastructure for the development of this study.

\section{REFERENCES}

[1] "Análise de dados das campanhas de prevenção ao câncer da pele promovidas pela Sociedade Brasileira 


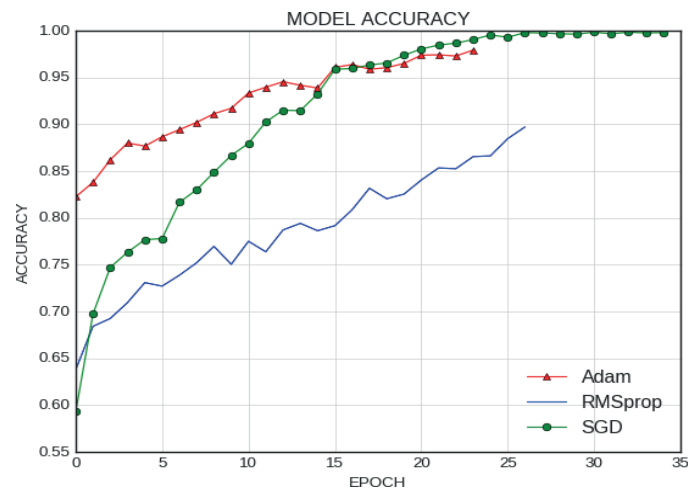

(a)

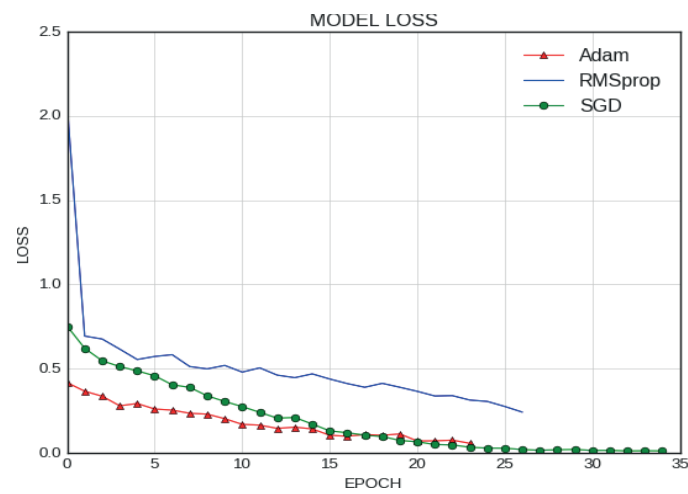

(b)

Fig. 3. Performance of the model with 2400 images of training. (a). Accuracy, (b). Loss

de Dermatologia de 1999 a 2005," pt, Anais Brasileiros de Dermatologia, vol. 81, pp. 533-539, Dec. 2006, ISSN: 0365-0596. [Online]. Available: http: / / www. scielo.br / scielo.php? script $=$ sci_arttext\& pid $=$ S0365 05962006000600004\&nrm=iso.

[2] C. M. Balch, "Cutaneous melanoma.," Annals of Surgical Oncology, 1992.

[3] W. Clark, L. From, E. Bernardino, and M. Mihm, "The histogenesis and biologic behavior of primary human malignant melanomas of the skin," Cancer Research, vol. 29, no. 3, pp. 705-727, Mar. 1969, ISSN: 00085472. [Online]. Available: http://europepmc.org/ abstract/MED/5773814.

[4] J. Scotto and T. R. Fears, "The association of solar ultraviolet and skin melanoma incidence among caucasians in the united states," Cancer Investigation, vol. 5, no. 4, pp. 275-283, 1987, PMID: 3664331. DOI: 10.1080/07357908709170100. eprint: https://doi.org/10.1080/07357908709170100.

[Online]. Available: https:/doi. org/10.1080/07357908709170100.

[5] N. Razmjooy, V. V. Estrela, H. J. Loschi, and W. Farfan, "A comprehensive survey of new meta-heuristic algorithms," Recent Advances in Hybrid Metaheuristics for Data Clustering, 2019.

[6] N. Razmjooy, M. Ashourian, V. V. Estrela, and H. J. Loschi, "Entropy-based breast cancer detection in digital mammograms using world cup optimization al- gorithm," International Journal of Swarm Intelligence Research (IJSIR), 2019, ISSN: 1947-9263.

[7] N. Razmjooy, M. Ashourian, V. V.Estrela, H. J. Loschi, R. P. França, and M. Vishnevski, "Computer-aided diagnosis of skin cancer: A review," Current Medical Imaging Reviews, 2019, ISSN: 1573-4056 (Print).

[8] N. Razmjooy and V. V. Estrela, "Applications of image processing and soft computing systems in agriculture," 2019. DOI: $10.4018 / 978-1-5225-8027-0$.

[9] D. J. Hemanth and V. V. Estrela, Deep Learning for Image Processing Applications, ser. Advances in Parallel Computing. IOS Press, 2017, ISBN: 9781614998228. [Online]. Available: https://books.google.com.br/books? $\mathrm{id}=\mathrm{vsFVDwAAQBAJ}$.

[10] I. Goodfellow, Y. Bengio, and A. Courville, Deep Learning. MIT Press, 2016, http://www. deeplearningbook.org.

[11] L. Deng and D. Yu, "Deep learning: Methods and applications," Found. Trends Signal Process., vol. 7, no. 3\&\#8211;4, pp. 197-387, Jun. 2014, ISSN: 19328346. DOI: 10.1561/2000000039. [Online]. Available: http://dx.doi.org/10.1561/2000000039.

[12] C. Dong, C. C. Loy, K. He, and X. Tang, "Learning a deep convolutional network for image super-resolution," in Computer Vision - ECCV 2014, D. Fleet, T. Pajdla, B. Schiele, and T. Tuytelaars, Eds., Cham: Springer International Publishing, 2014, pp. 184-199, ISBN: 978- 3-319-10593-2.

[13] M. A. Nielsen, Neural networks and deep learning, misc, 2018. [Online]. Available: http:// neuralnetworksanddeeplearning.com/.

[14] G. J. S. Litjens, T. Kooi, B. E. Bejnordi, A. A. A. Setio, F. Ciompi, M. Ghafoorian, J. A. W. M. van der Laak, B. van Ginneken, and C. I. Sánchez, "A survey on deep learning in medical image analysis.," Medical Image Analysis, vol. 42, pp. 60-88, 2017. [Online]. Available: http://dblp.uni- trier.de/db/journals/mia/mia42.html\# LitjensKBSCGLGS17.

[15] Y. Lv, Y. Duan, W. Kang, Z. Li, and F.-Y. Wang, "Traffic flow prediction with big data: A deep learning approach," IEEE Transactions on Intelligent Transportation Systems, vol. 16, pp. 865-873, 2015.

[16] Y. Chen, Z. Lin, X. Zhao, G. Wang, and Y. Gu, "Deep learning-based classification of hyperspectral data," IEEE J. Sel. Topics in Applied Earth Observations and Remote Sensing, vol. 7, no. 6, pp. 2094-2107, Jun. 2014, ISSN: 1939-1404. DOI: 10.1109/JSTARS.2014. 2329330.

[17] N. Papernot, P. McDaniel, S. Jha, M. Fredrikson, Z. B. Celik, and A. Swami, "The limitations of deep learning in adversarial settings," in 2016 IEEE European Symp. on Sec. and Priv. (EuroS P), Mar. 2016, pp. 372-387. DOI: 10.1109/EuroSP.2016.36.

[18] I. Arel, D. Rose, and T. Karnowski, "Deep machine learning - a new frontier in artificial intelligence research [research frontier].," IEEE Comp. Int. Mag., vol. 5, pp. 13-18, Jan. 2010. 
[19] D. Shen, G. Wu, and H.-I. Suk, "Deep learning in medical image analysis," Annual Review of Biomedical Engineering, vol. 19, Mar. 2017. DOI: 10.1146/annurev- bioeng-071516-044442.

[20] Y. Bengio, "Learning deep architectures for ai," Found. Trends Mach. Learn., vol. 2, no. 1, pp. 1-127, Jan. 2009, ISSN: $\quad 1935-8237 . \quad$ DOI: $10.1561 / 2200000006$. [Online]. Available: http://dx.doi.org/10.1561/2200000006.

[21] V. Mnih, K. Kavukcuoglu, D. Silver, A. A. Rusu, J. Veness, M. G. Bellemare, A. Graves, M. Riedmiller, A. K. Fidjeland, G. Ostrovski, S. Petersen, C. Beattie, A. Sadik, I. Antonoglou, H. King, D. Kumaran, D. Wierstra, S. Legg, and D. Hassabis, "Human-level control through deep reinforcement learning," Nature, vol. 518, no. 7540, pp. 529-533, Feb. 2015, ISSN: 00280836. [Online]. Available: http://dx.doi.org/10.1038/nature14236.

[22] W. Samek, T. Wiegand, and K. Müller, "Explain- able artificial intelligence: Understanding, visualizing and interpreting deep learning models," CoRR, vol. abs/1708.08296, 2017. arXiv: 1708.08296. [On- line]. Available: http://arxiv.org/abs/1708.08296.

[23] F. Pedregosa, G. Varoquaux, A. Gramfort, V. Michel, B. Thirion, O. Grisel, M. Blondel, P. Prettenhofer,

R. Weiss, V. Dubourg, J. Vanderplas, A. Passos, D. Cournapeau, M. Brucher, M. Perrot, and É Duchesnay, "Scikit-learn: Machine learning in python," J. Mach. Learn. Res., vol. 12, pp. 2825-2830, Nov. 2011, ISSN: 1532-4435.

[Online].

Available: http://dl.acm.org/citation.cfm?id=1953048.2078195.

[24] T. E. Oliphant, "Python for scientific computing," Computing in Science Engineering, vol. 9, no. 3, pp. 10-20, May 2007, ISSN: 1521-9615. DOI: 10.1109/MCSE. 2007.58

[25] M. F. Sanner, "Python: A programming language for software integration and development.," Journal of Molecular Graphics Modelling, vol. 17 1, pp. 57-61, 1999.

[26] F. Sebastiani, "Machine learning in automated text categorization," ACM Comput. Surv., vol. 34, no. 1, pp. 1-47, Mar. 2002, ISSN: 0360-0300. DOI: 10.1145/ 505282.505283. [Online]. Available: http://doi.acm.org/ $10.1145 / 505282.505283$.

[27] I. H. Witten and E. Frank, Data Mining: Practical Machine Learning Tools and Techniques, 2nd, ser. Morgan Kaufmann Series in Data Management Systems. Morgan Kaufmann, 2005.

[28] M. Abadi, P. Barham, J. Chen, Z. Chen, A. Davis, J. Dean, M. Devin, S. Ghemawat, G. Irving, M. Isard, M. Kudlur, J. Levenberg, R. Monga, S. Moore, D. G Murray, B. Steiner, P. Tucker, V. Vasudevan, P.Warden, M. Wicke, Y. Yu, and X. Zheng, "Tensorflow: A system for large-scale machine learning," in 12th USENIX Symp. on Op. Systems Des. and Implem. (OSDI 16), 2016, pp. 265-283. [Online]. Available: https://www. usenix.org/system/files/conference/osdi16/osdi16-abadi. pdf.
[29] E. Alpaydin, Introduction to Machine Learning, 2nd. The MIT Press, 2010, ISBN: 026201243X, 9780262012430.

[30] J. Deng, W. Dong, R. Socher, L. Li, Kai Li, and Li FeiFei, "Imagenet: A large-scale hierarchical image database," in 2009 IEEE Conference on Computer Vision and Pattern Recognition, Jun. 2009, pp. 248255. DOI: 10.1109/CVPR.2009.5206848.

[31] C. N. Vasconcelos and B. N. Vasconcelos, "Increasing deep learning melanoma classification by classical and expert knowledge based image transforms," CoRR, vol. abs/1702.07025, 2017. arXiv: 1702.07025. [On- line]. Available: http://arxiv.org/abs/1702.07025.

[32] H. Roth, A. Farag, L. Lu, E. B. Turkbey, and R. M. Summers, "Deep convolutional networks for pancreas segmentation in CT imaging," CoRR, vol. abs/1504.03967, 2015. arXiv: 1504.03967. [On- line]. Available: http://arxiv.org/abs/1504.03967.

[33] M. Melinscak, P. Prentasic, and S. Loncaric, "Retinal vessel segmentation using deep neural networks," VISAPP 2015 - 10th International Conference on Computer Vision Theory and Applications; VISIGRAPP, Proceedings, vol. 1, pp. 577-582, Jan. 2015. DOI: 10.5220/ 0005313005770582.

[34] M. Havaei, A. Davy, D. Warde-Farley, A. Biard, A. C. Courville, Y.Bengio, C. Pal, P. Jodoin, and H. Larochelle, "Brain tumor segmentation with deep neural networks," CoRR, vol. abs/1505.03540, 2015. arXiv: 1505.03540. [Online]. Available: http://arxiv. org/abs/1505.03540.

[35] S. Pereira, A. Pinto, V. Alves, and C. Silva, "Deep convolutional neural networks for the segmentation of gliomas in multi-sequence mri," vol. 9556, Jan. 2016, pp. 131-143, ISBN: 978-3-319-30857-9. DOI: 10.1007/ 978-3-319-30858-6 12 .

[36] A. Krizhevsky, I. Sutskever, and G. E. Hinton, "Imagenet classification with deep convolutional neural networks," Neural Information Processing Systems, vol. 25, Jan. 2012. DOI: 10.1145/3065386.

[37] I. porject, Public skin lesion image archive, https://www.isic-archive.com/\#!/topWithHeader/ onlyHeaderTop/gallery, 2018.

[38] H. Wu and X. Gu, "Max-pooling dropout for regularization of convolutional neural networks," CoRR, vol. abs/1512.01400, 2015. arXiv: 1512.01400. [Online]. Available: http://arxiv.org/abs/1512.01400.

[39] S. Ioffe and C. Szegedy, "Batch normalization: Accelerating deep network training by reducing internal covariate shift," CoRR, vol. abs/1502.03167, 2015. arXiv: 1502.03167. [Online]. Available: http://arxiv.org/abs/ 1502.03167.

[40] A. Gron, Hands-On Machine Learning with ScikitLearn and TensorFlow: Concepts, Tools, and Techniques to Build Intelligent Systems, 1st. O'Reilly Media, Inc., 2017, ISBN: 1491962291,9781491962299.

[41] S. Ruder, "An overview of gradient descent optimization algorithms," CoRR, vol. abs/1609.04747, 2016. 
arXiv: 1609.04747. [Online]. Available: http://arxiv. org/abs/1609.04747.

[42] K. Janocha and W. M. Czarnecki, "On loss functions for deep neural networks in classification," CoRR, vol. abs/1702.05659, 2017. arXiv: 1702.05659. [On- line]. Available: http://arxiv.org/abs/1702.05659.

[43] F. Chollet et al., Keras, https://keras.io, 2015.

[44] J. D. Hunter, "Matplotlib: A 2d graphics environment," Computing in Science \& Engineering, vol. 9, no.3, pp. 90-95, 2007. DOI: 10.1109/MCSE.2007.55.

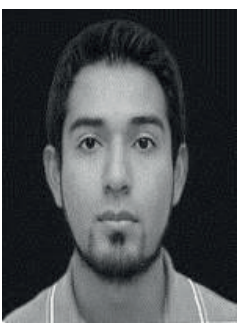

Pablo Minango. B.Sc. in Electronic Engineering from the Universidad Politécnica Salesiana (UPS), Quito, Ecuador, in 2017. Currently, he is an M.Sc. candidate by Department of Communications, Faculty of Electrical and Computer Engineering (FEEC) at State University of Campinas UNICAMP. His research interests include Deep Learning, Machine Learning, Digital Image Processing with Medicals images.

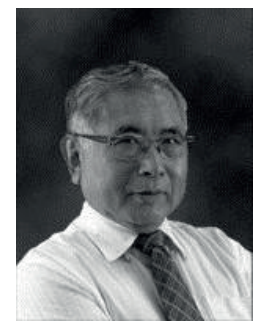

Yuzo Iano. B.Sc. (1972), M.Sc. (1974) and Ph.D. degrees (1986) in Electrical Eng. at UNICAMP, Brazil. He has been working in the technological production field, with 1 patent granted, 8 filed patent applications and 36 projects completed with research and development agencies. He has supervised 29 doctoral theses, 49 master's dissertations, 74 undergraduate and 48 scientific initiation works. He has participated in more than 100 master's examination boards, 50 doctoral degrees, author of 2 books and more than 250 published articles. He is currently

Professor at UNICAMP, Editor-in-Chief of the SET International Journal of Broadcast Engineering and General Chair of the Brazilian Symposium on Technology (BTSym). He has experience in Electrical Engineering, with knowledge in Telecommunications, Electronics and Information Technology, mainly in the field of audio-visual communications and multimedia.

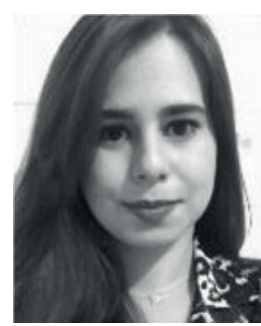

Ana Carolina Borges Monteiro. Graduated in Biomedicine from Centro Universitário AmparenseUNIFIA (2015). Currently, she pursues a Ph.D. Candidate by Department of Communications (DECOM), Faculty of Electrical and Computer Engineering (FEEC) at State University of Campinas (UNICAMP), and a researcher at the Laboratory of Visual Communications (LCV). She is also the Registration Chair of the Brazilian Symposium on Technology (BTSym) and has expertise in the areas of Clinical Analysis and digital image processing

through MATLAB software. She has performed work, research experiments/projects, and internship in municipal hospital, and works as a reviewer.

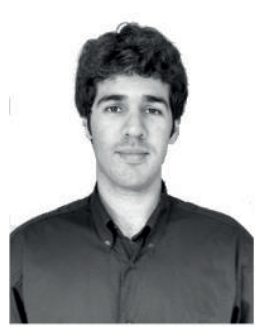

Reinaldo Padilha França. B.Sc. in Computer Engineering from University Regional Center of Espírito Santo de Pinhal - 2014. Presently, he is a $\mathrm{Ph} . \mathrm{D}$. Candidate by Department of Communications (DECOM), Faculty of Electrical and Computer Engineering (FEEC) at State University of Campinas (UNICAMP), and a researcher at the Laboratory of Visual Communications (LCV). He is also the Proceedings Chair of the Brazilian Symposium on Technology (BTSym). His interest includes programming and development in $(\mathrm{C} / \mathrm{C}$ ++ , Java and .NET

languages). Simulation, Operating Systems, Software Engineering, Wireless and Network, Internet of Things, Broadcasting, Image Processing, Multimedia, and Telecommunications Systems.

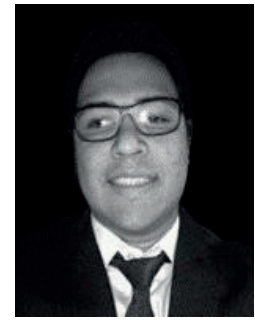

Gabriel Gomes de Oliveira. B.Sc. in Civil Engineering from the UNIP University in 2018. He is pursuing a master's degree at UNICAMP, Faculty of Electrical and Computer Engineering (FEEC), Communications Department (DECOM), Laboratory of Visual Communication (LCV). Currently, he researches and studies the area Smart Cities, Internet of Things, environmental engineering and cyber-physical systems. 